\title{
The effect of a novel probiotic on metabolic biomarkers in adults with prediabetes and recently diagnosed type 2 diabetes mellitus: study protocol for a randomized controlled trial
}

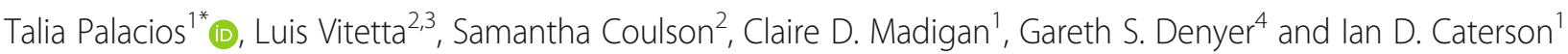

\begin{abstract}
Background: Shifts in the gastrointestinal microbiome have been shown to contribute to the progression of metabolic diseases including prediabetes and type 2 diabetes mellitus. Research suggests that in-vivo modulation of the gut microbiome by specific probiotic microorganisms may improve insulin sensitivity and blood sugar management, preventing or delaying the development of type 2 diabetes mellitus. However, further research is needed to understand the effect of probiotics as a therapy for the treatment of metabolic diseases. An evidence-based multi-species probiotic was developed to encourage a shift in the gastrointestinal bacterial cohort from a disease-prone to a balanced state with the aim of improving metabolic markers associated with type 2 diabetes mellitus.

Methods: Sixty adults with a body mass index $\geq 25 \mathrm{~kg} / \mathrm{m}^{2}$ with prediabetes or type 2 diabetes mellitus (diagnosed within the previous 12 months) will be enrolled in a double-blind, placebo-controlled pilot study. Participants will be randomized to a multi-species probiotic or placebo for 12 weeks. Both groups will receive lifestyle and nutritional advice. The primary outcome measure is the change between groups in fasting plasma glucose levels from baseline to 12 weeks. Secondary outcome measures include, but are not limited to, the change in lipid profile, systemic inflammation, gut permeability, and faecal microbial and metabolomic profiles. Blood and stool samples are collected at baseline and 12 weeks after treatment.
\end{abstract}

Discussion: Intentional manipulation of gastrointestinal microbial profiles may be useful for preventing and controlling type 2 diabetes mellitus and its associated metabolic complications.

Trial registration: Australian New Zealand Clinical Trials Registry, ACTRN12613001378718. Registered on 16 December 2013

Keywords: Gut microbiota, Prediabetes, Probiotics, Type 2 diabetes mellitus

\footnotetext{
* Correspondence: talia.palacios@sydney.edu.au

${ }^{1}$ The Boden Institute of Obesity, Nutrition, Exercise and Eating Disorders,

University of Sydney, Sydney, Australia

Full list of author information is available at the end of the article
} 


\section{Background}

The relationship between the gastrointestinal microbiota and obesity-associated disorders has gained extensive research interest in the past 10 years. A disturbed gut microbiota expressed as gut dysbiosis (an intestinal physical barrier abnormality) has been associated with the progression and maintenance of obesity, type 2 diabetes mellitus, cardiovascular diseases and metabolic syndrome [1-5]. The mechanisms by which gut dysbiosis produces and influences these metabolic alterations are via regulation of the host's energy balance and storage and by promoting endotoxaemia or bacteraemia [6]. Furthermore, postprandial blood glucose levels are very much influenced by the gut bacteria, with recent research demonstrating a profound influence on how individuals responded to identical food items that could be accurately predicted based on their gut bacterial profiles [7]. Gut dysbiosis may be restored to a balanced state through microbiota-based interventions, which may improve metabolic markers associated with type 2 diabetes mellitus through immunomodulatory and anti-inflammatory pathways.

A probiotic is a microbiota-based intervention defined as 'a live microorganism, which, when administered in adequate amounts, confers beneficial health effects on the host' $[2,8,9]$. A meta-analysis evaluating the effect of probiotics on glycaemia suggests that probiotics can play an important role in the prevention and treatment of type 2 diabetes mellitus [10]. Certain probiotic species have improved insulin sensitivity, inflammatory markers and lipid profiles in obese, type 2 diabetes mellitus and dyslipidaemic subjects [11-13]. However, it is not known whether a combination of probiotic species that have demonstrated beneficial therapeutic effects individually can improve metabolic markers associated with type 2 diabetes mellitus and have an additive effect to standard care. Therefore, we have formulated a novel prescription containing eight probiotic species that belong to the Lactobacillus, Bifidobacterium, Streptococcus and Saccharomyces genera to improve glucose metabolism in subjects with prediabetes and early type 2 diabetes mellitus. This multi-species probiotic formula has been tested previously in our laboratory using in-vitro models with rodent fat and muscle cell lines. The results from these in-vitro experiments showed the supernatants collected from the growth media of the probiotic decreased lipid accumulation in 3T3-L1 adipocytes and restored glucose uptake in insulin resistant L6 muscle cells [7]. The formulation and dosage proposed in this study have not been investigated previously in human studies. Therefore, the aim is to test the safety and efficacy of this novel probiotic formulation in adults with prediabetes and early type 2 diabetes mellitus. We hypothesize that a shift in the gut microbiome induced by this multi-species probiotic will decrease metabolic and inflammatory markers and result in improved blood glucose management.

\section{Methods \\ Design}

This pilot study is a single site, randomized, doubleblind, placebo-controlled clinical trial conducted at the Charles Perkins Centre Royal Prince Alfred Clinic in Sydney, Australia. Sixty adults with prediabetes or early type 2 diabetes mellitus will be randomized to take either a multi-species probiotic capsule or placebo for 12 weeks. The Standard Protocol Items: Recommendations for Interventional Trials (SPIRIT) was used to elaborate the study protocol (see Additional file 1). Participants' progression through the trial is presented in Fig. 1 (CONSORT diagram [14]).

\section{Recruitment process}

Subject recruitment will be through the Boden Institute's clinical trials register, the Sydney Local Health District intranet, the University of Sydney website and Diabetes Australia social media channels.

\section{Allocation}

Participants will be randomized to the probiotic or placebo group without stratification using computer-generated random numbers (FileMarker Pro). Both participants and study investigators will be blinded to treatment allocation. Participant unblinding will only be requested in a medical emergency, where knowledge of the study treatment is essential for any treatment of the participant. The reason for unblinding will be documented and the study treatment will not be revealed to any member of the study team.

\section{Data handling and record keeping}

Data is completed on case report forms, source documents (both written on paper and time and date stamped electronic capture) and entered into a database. This database will be password protected and backed up the University of Sydney server. All results will be sent to participants by email.

\section{Inclusion criteria}

Participants are eligible for the study if they meet the following criteria:

- Aged $\geq 18$ years

- Body mass index $\geq 25 \mathrm{~kg} / \mathrm{m}^{2}$

- Have prediabetes or have been diagnosed with type 2 diabetes mellitus within the previous 12 months

- Are treated by diet alone or diet plus metformin 


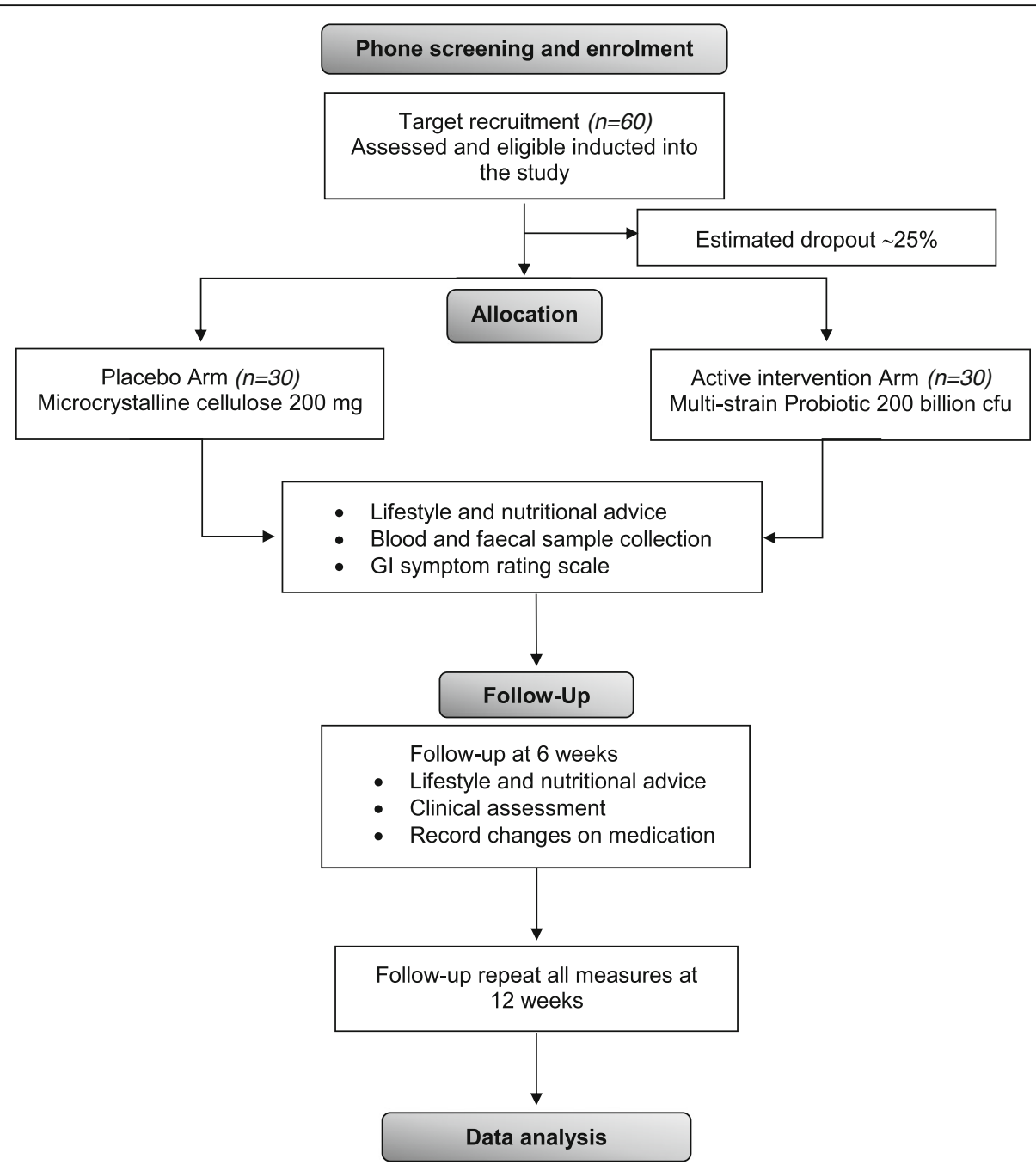

Fig. 1 CONSORT flowchart of participants' progress through the study

- Are willing to adhere to the study protocol (no yoghurt, fermented food, dietary supplements, probiotics or prebiotics) for the duration of the study.

The definition of prediabetes is based on the American Diabetes Association guidelines [15] and participants must have: (i) a fasting plasma glucose level between $5.6 \mathrm{mmol} / \mathrm{l}$ and $6.9 \mathrm{mmol} / \mathrm{l}$ or; (ii) two-hour post-challenge (oral glucose tolerance test) plasma glucose level between $7.8 \mathrm{mmol} / \mathrm{l}$ and $11 \mathrm{mmol} / \mathrm{l}$ or; (iii) $\mathrm{HbAlc}$ between $5.7 \%$ and $6.4 \%$.

The criteria for the diagnosis of type 2 diabetes mellitus is based on the American Diabetes Association guidelines [15]. Participants are included in the study if they have been diagnosed with type 2 diabetes mellitus by their general practitioner in the previous 12 months or have: (i) a fasting plasma glucose level $\geq 7.0 \mathrm{mmol} / \mathrm{l}$ or; (ii) 2-hour post-challenge (oral glucose tolerance test) plasma glucose level $\geq 11.1 \mathrm{mmol} / \mathrm{l}$ or; (iii) an HbA1c $\geq 6.5 \%$.

\section{Exclusion criteria}

- Type 1 diabetes mellitus

- Type 2 diabetes mellitus diagnosed for longer than 12 months

- Taking anti-obesity drugs or blood glucose-lowering medications (i.e. sulfonylureas, alpha-glucosidase inhibitors, thiazolidinediones and glucagon-like peptide-1 analogues) other than metformin

- Concomitant gastrointestinal disorders (i.e., irritable bowel syndrome, inflammatory bowel disease and coeliac disease)

- Recent use (within the previous 4 weeks) of antibiotics and dietary supplements (fish oil, probiotics, prebiotics, multivitamins, minerals, nutraceuticals and herbal preparations)

- Pregnancy, breastfeeding or planning to become becoming pregnant

- Alcohol abuse or the use of any illicit drugs 
- Clinical evidence of active infection or any severe illness unrelated to diabetes.

\section{Intervention and compliance Investigational product}

The study coordinator will obtain written informed consent and randomize eligible participants to the multispecies probiotic or placebo. The study product contains either $50 \times 10^{9}$ colony forming units (cfu) of a multispecies probiotic $\left(27 \times 10^{9}\right.$ cfu of Lactobacillus spp., $22.5 \times 10^{9} \mathrm{cfu}$ of Bifidobacterium spp., $450 \times 10^{6} \mathrm{cfu}$ of Streptococcus spp. and $45 \times 10^{6} \mathrm{cfu}$ of Saccharomyces spp., patent pending) or $50 \mathrm{mg}$ of the placebo (maltodextrin) per capsule. The probiotic and placebo capsules are opaque white and look and smell identical. Verbal and written instructions on how to take the study product or placebo will be provided at the initial and each review visit. Enrolled participants will be required to:

- Take two capsules twice per day (20 min before breakfast and dinner) with cold non-carbonated water. The product is not to be mixed or taken with hot drinks or foods, as heat and stomach acids can reduce the stability of the probiotic bacteria

- Store the study product in the fridge at $4-6{ }^{\circ} \mathrm{C}$

- Record the number of capsules taken each day in the study diary

- Bring all capsules remaining in the bottle to the next visit

- Avoid eating or drinking yoghurt, fermented food, dietary supplements (i.e. vitamins, minerals, nutraceuticals, herbal preparations, probiotics, prebiotics or fish oils) and antibiotics (unless recommended by a health professional).

\section{Study product compliance}

Capsule counting, at weeks 6 and 12, will be used to assess the participant's compliance in taking the study product. A record of the date, the visit and the amount of study product dispensed and returned will be documented for each participant. Participants are defined as non-compliant if they have taken less than $80 \%$ of the study product on both occasions.

\section{Lifestyle guidance}

Subjects in both groups of the study will receive dietary and lifestyle advice from a research dietitian. This advice is according to the 2013 National Health and Medical Research Council dietary guidelines for Australian adults. The guidelines focus on improving fruit and vegetable intake, portion size control, reduction of energy-dense and low-nutrient density foods and drinks, encouraging physical activity by walking 10,000 steps per day and reducing sedentary behaviour. At baseline, participants will be provided with a pedometer to track the number of steps they take.

\section{Outcome measures \\ Primary outcome measure}

The difference in any change in fasting plasma glucose level between the intervention and placebo groups between baseline and 12 weeks.

\section{Secondary outcome measures}

- The safety of the probiotic formula

- The change between groups in the following biomarkers or measurements from baseline to 12 weeks:

(i) HbA1c

(ii)insulin sensitivity indices, including Matsuda-ISI [16] and homeostatic model assessment-IR [17]

(iii) fasting plasma lipids, such as triglycerides, free fatty acids, total cholesterol, HDL-c and LDL-c

(iv) plasma zonulin and lipopolysaccharide

(v) hs-CRP

(vi) gastrointestinal symptoms

(vii) body mass index, waist circumference, and waist and hip ratio

(viii)blood pressure

(ix) faecal microbial and metabolomics profiles.

\section{Participant timeline}

Screening, intervention and assessment visits will be performed by a research nutritionist. The schedule of visits and measurements is given in Fig. 2 in compliance with SPIRIT guidelines.

\section{Data collection}

\section{Clinical examination}

Medical history, prescribed and non-prescribed medications, alcohol intake and smoking habits will be recorded during the screening visit. Anthropometric measurements will be collected using standardized examination procedures [18] and calibrated equipment at three time points (baseline, 6 weeks and 12 weeks). Height will be measured using a stadiometer with an adjustable headpiece. Participants will be weighed wearing an examination gown and underwear only. Waist circumference will be measured at the midpoint between the lowest margin of the last rib and the top of the iliac crest. Hip circumference is measured around the widest portion of the buttocks.

Participants will record a 7 day food diary (5 working days and 2 weekend days) at baseline, then at 6 and 12 weeks after randomization. Food Works 8 will be used to assess energy and macronutrients including dietary fibre intake. Participants will wear a pedometer for the 


\begin{tabular}{|c|c|c|c|c|c|}
\hline \multirow[b]{3}{*}{ TIMEPOINT** } & \multicolumn{5}{|c|}{ STUDY PERIOD } \\
\hline & \multirow{2}{*}{$\begin{array}{c}\text { Enrolment } \\
-1 \text { week }\end{array}$} & \multirow{2}{*}{$\begin{array}{c}\text { Allocation } \\
0\end{array}$} & \multicolumn{3}{|c|}{ Post-allocation } \\
\hline & & & week 1 & Week 6 & Week 12 \\
\hline \multicolumn{6}{|l|}{ ENROLMENT: } \\
\hline \multirow{2}{*}{$\begin{array}{l}\text { Eligibility screen } \\
\text { Informed consent }\end{array}$} & $x$ & & & & \\
\hline & $X$ & & & & \\
\hline Medical history & $x$ & & & & \\
\hline Allocation & & $x$ & & & \\
\hline \multicolumn{6}{|l|}{ INTERVENTIONS: } \\
\hline \multicolumn{6}{|l|}{ Probiotic capsules } \\
\hline \multicolumn{6}{|l|}{ Placebo capsules } \\
\hline \multicolumn{6}{|l|}{ ASSESSMENTS: } \\
\hline $\begin{array}{c}\text { Anthropometric } \\
\text { measurements }\end{array}$ & $x$ & & $\leftarrow$ & & \\
\hline Blood test & & & $x$ & & $x$ \\
\hline $\begin{array}{r}\text { Stool sample } \\
\text { collection }\end{array}$ & & & $X$ & & $\mathrm{X}$ \\
\hline \multicolumn{6}{|l|}{ Physical activity } \\
\hline $\begin{array}{r}\text { Food diary } \\
\text { analysis }\end{array}$ & & & X & $\mathrm{X}$ & $\mathrm{X}$ \\
\hline $\begin{array}{r}\text { GI symptom rating } \\
\text { scale }\end{array}$ & & & $x$ & $X$ & $x$ \\
\hline $\begin{array}{r}\text { Adverse events } \\
\text { monitoring }\end{array}$ & & & & & \\
\hline
\end{tabular}

Fig. 2 SPIRIT schedule of enrolment, interventions and assessments

study duration. The Stanford Leisure-Time Activity Categorical Item (L-Cat) 2.2 physical activity questionnaire [19] will be completed at baseline, and 6 weeks and 12 weeks after treatment. The number of steps taken will be recorded daily in a study diary.

\section{Blood samples}

Blood samples will be collected after fasting overnight at baseline and 12 weeks. Participants who do not have type 2 diabetes mellitus will undergo a $75 \mathrm{~g}$ oral glucose tolerance test to assess glucose and insulin levels at three time points $(0 \mathrm{~min}, 60 \mathrm{~min}$ and $120 \mathrm{~min}$ ). Blood samples to measure glucose, insulin, HbAlc, lipids and hs-CRP will be sent to a commercial laboratory for analysis. Samples of $12 \mathrm{ml}$ of blood collected in ethylenediaminetetraacetic acid (EDTA) plasma tubes will be used for lipopolysaccharide and zonulin analysis. These samples will be immediately placed at $4{ }^{\circ} \mathrm{C}$ and centrifuged for 15 minutes at $1000 \mathrm{~g}$ (or $3000 \mathrm{rpm}$ ) at $2-8{ }^{\circ} \mathrm{C}$ within
30 minutes. Plasma samples will be aliquoted in pyrogenfree tubes using pyrogen-free pipette tips and stored at $-80{ }^{\circ} \mathrm{C}$. Serum endotoxin will be measured using the Pierce $^{\mathrm{Tn}}$ Limulus Amoebocyte Lysate chromogenic endotoxin quantification kit (Thermo Fisher, Sydney, NSW, Australia). The EDTA-plasma zonulin concentration will be measured with the human haptoglobin ELISA kit (Abcam ${ }^{\circ}$, Sydney, NSW, Australia). Lipopolysaccharide and zonulin analyses will be performed in duplicate.

\section{Stool samples}

Stool samples will be collected at baseline and 12 weeks using a stool specimen collection kit. This collection kit includes an instruction booklet for the stool sample collection and transportation, ice packs, gloves, a sterile container, sealed plastic pouch, cool box and an AnaeroGen ${ }^{\mathrm{nt}}$ Compact sachet, which preserves the microbiological characteristics of the sample for 72 hours. Participants will bring 
the stool sample to the clinic and the containers will be stored at $4{ }^{\circ} \mathrm{C}$ and analyzed by matrix-assisted laser desorption ionization time-of-flight mass spectrometry 24-48 hours after collection. In addition, a $1 \mathrm{~g}$ sample will be stored at $-80{ }^{\circ} \mathrm{C}$ for faecal microbial and metabolite profiling. DNA extraction will be performed using the QIAamp DNA stool kit (Qiagen, Sydney, NSW, Australia). Faecal microbial composition will be identified by sequencing the 16S rRNA using the Ion S5 next-generation sequencing system (Thermo Fisher, Sydney, NSW, Australia). Shortchain fatty acids, secondary bile acids and choline byproducts will be measured using gas chromatography-mass spectrometry.

\section{Data analysis and statistical considerations Sample size}

As this is a pilot study, no evidence about blood glucose differences is available for this product. Therefore, it was decided to use the number of participants needed to detect a mean difference between the groups in fasting plasma glucose level of $2.0 \mathrm{mmol} / \mathrm{l}$ (standard deviation $2.0 \mathrm{mmol} / \mathrm{l}$ ) at week 12 with $80 \%$ power and $95 \%$ confidence as a starting point. The number of subjects needed is 32 . The fact that the study includes two different cohorts (participants with prediabetes and early type 2 diabetes mellitus) was also taken into consideration, as owing to the lack of stratification at randomization the variance could be high. Therefore, the sample size has been inflated to 30 per group (including a drop-out rate of $25 \%$ ) to give a total sample size of 60 eligible participants.

\section{Statistical analysis}

Analysis will be conducted using the intention-to-treat principle and missing data will be imputed with baseline values for a conservative estimate (i.e. no change). Descriptive statistics will be presented as mean \pm standard deviation, or median with range, as appropriate. Primary and secondary outcomes will be analyzed using generalized linear models. The following covariates will be added to the model: sex, age and percentage weight change. Additionally, if there is an imbalance between the groups of prediabetes and type 2 diabetes mellitus, this will be included as a covariate in the model. Three planned subgroup analyses will be completed between participants who are taking metformin, participants classified as prediabetic or type 2 diabetes mellitus and participants defined as compliant. Chi-square analysis will determine associations between categorical variables.

For the gut microbiome analysis, sequenced data will be interpreted using the bioinformatics tools programmed in the Ion Reporter ${ }^{\mathrm{TM}}$ software. Quantitative Insights Into Microbial Ecology (QIIME) algorithms will determine the bacterial diversity within a sample (alpha diversity) and between all the samples (beta diversity). Additionally, multivariate data analysis with principal component analysis on the diversity indexes and comparisons of genus and species level data will be performed to reveal differences in the microbial composition between the two groups. Differences in relative abundance of taxa between the intervention and placebo group and between participants receiving and not receiving metformin will be determined using ANOVA, using subject as a blocking factor. Changes in relative abundance will be tested for correlations with patient biochemical measurements. For all statistical tests, the Benjamini-Hochberg false discovery rate adjustment [20] will be used to account for the number of taxa tested in each comparison.

\section{Adverse events}

Adverse events are defined as any unfavourable and unintended sign (including an abnormal laboratory finding), symptom, or disease (new or exacerbated) temporally associated with the use of the study product, whether or not considered related to the treatment. The study investigator will monitor each participant for adverse events during the study. All adverse signs or symptoms reported between consent and final follow-up will be recorded. Adverse events are reported descriptively by group.

\section{Serious adverse events}

All serious adverse events, related or not related to the study product, are recorded on paper and electronic case report forms. Serious adverse events will be reported in compliance with the requirements of the Sydney Local Health District Human Research Ethics Committee. Probiotic intake has not been associated with any major side effects and extensive safety data are available on their effects; however, participants will be discontinued from the study product if it is decided that a serious adverse event may be related to probiotic consumption.

\section{Ethical considerations}

Ethical approval has been granted by the Sydney Local Health District Human Research Ethics Committee (Royal Prince Alfred Hospital). This study will be carried out according to the Declaration of Helsinki, the National Health and Medical Research Council National Statement on Ethical Conduct in Research Involving Humans and the Notes for Guidance on Good Clinical Practice as adopted by the Australian Therapeutic Goods Administration (2000) (CPMP/ICH/135/95) and the International Conference on Harmonisation Good Clinical Practise guidelines.

\section{Discussion}

This randomized trial will assess the efficacy and safety of a multi-species probiotic formulation in the management 
of prediabetes and type 2 diabetes mellitus metabolic markers. The study outcomes may lead to novel treatments to reduce the metabolic disturbances associated with these disorders. This study will also provide empirical evidence to address currently unresolved issues with the efficacy and safety of probiotics. In designing this clinical study, several key decisions were made to overcome current limitations in the published literature and reduce possible biases.

While focusing on those with prediabetes, subjects with early type 2 diabetes mellitus were included to assess the feasibility of inducing partial or complete remission of type 2 diabetes mellitus. This decision also meant including participants taking metformin as this drug is commonly prescribed early in type 2 diabetes mellitus; it has also been shown to have effects on the microbiome [21, 22]. Furthermore, microbiota-based interventions may reduce gastrointestinal symptoms associated with metformin administration with a consequent improvement in medication compliance [23]. As far as we are aware, the interaction between the gastrointestinal microbiota, probiotics and metformin has not been explored in patients with prediabetes and type 2 diabetes mellitus. Therefore, this study will assess the efficacy and safety of a novel multi-species probiotic and provide preliminary data on its effect on metformin.

Limitations of the study include the short-term impact of the intervention, as subjects will be treated for only 12 weeks. Additionally, the small number of visits during the study can be considered a risk factor for noncompliance. Moreover, it must be remembered this study is investigating the short-term impact of a multi-species probiotic formulation to help improve fasting blood glucose levels. It is known that probiotics do not recolonize the intestinal tract but rather are transient colonizers and that wash-out of administered doses can take from approximately 4-6 weeks, thereby leading to a therapy that may be not be permanent.

This trial will provide pilot data of a novel probiotic formula that may shift the gastrointestinal microbial profile from a disease-prone to a balanced state and improve glucose metabolism. The findings will enhance the understanding of the role that probiotics may play on metabolic biomarkers in individuals with high glucose levels. Additionally, microbiome biomarkers associated with the risk of developing type 2 diabetes mellitus may be found by exploring the gut microbial and metabolomic profiles.

\section{Trial status}

Participant recruitment started in August 2015 and is ongoing.

\section{Additional files}

Additional file 1: SPIRIT 2013 checklist. (DOCX $51 \mathrm{~kb}$ )

Additional file 2: Participant information sheet and consent form for the study 'Effect of a novel probiotic on metabolic biomarkers in adults with prediabetes and recently diagnosed with type 2 diabetes'. (DOCX $71 \mathrm{~kb}$ )

\section{Abbreviations \\ ANOVA: analysis of variance; cfu: colony forming unit; EDTA: ethylenediaminetetraacetic acid; ELISA: enzyme-linked immunosorbent assay; HbA1c: glycosylated haemoglobin; hs-CRP: high-sensitive C-reactive protein; L-CAT: Stanford Leisure-Time Activity Categorical Item; QIIME: Quantitative Insights Into Microbial Ecology; SPIRIT: Standard Protocol Items: Recommendations for Interventional Trials}

\section{Acknowledgements}

The authors gratefully acknowledge Medlab Clinical for providing the study product and resources for sample analysis; Bioscreen Medical Laboratory, who will provide the stool sampling kits, process the faecal samples and perform the matrix-assisted laser desorption ionization time-of-flight mass spectrometry analysis; and Ms Alice Gibson, who designed the food diary booklet.

\section{Funding}

Funding is provided by Medlab Clinical Ltd. TP has received a scholarship from the Ecuadorian government (Secretaria de Educacion Superior, Ciencia, Tecnologia e Innovacion).

\section{Availability of data and materials}

Not applicable.

\section{Authors' contributions}

IDC is the principal investigator and will oversee the project implementation. IDC, LV, SC and TP designed the trial and wrote the study proposal, with input and advice from CDM. GSD developed the database and randomization tool. CDM wrote the statistical analysis plan with assistance from the other authors. All authors have read and approved the final version of the protocol.

\section{Competing interests}

This study is part of a PhD project supported by the University of Sydney, the Ecuadorian government and Medlab Clinical Ltd. (which produces the probiotic). LV has received National Institute of Complementary Medicine and National Health and Medical Research Council of Australia grant funding and industry support (FitBioceuticals Ltd, Swisse-Swisse Wellness and Parmalat) for research into probiotics. LV participates in research on probiotics at Medlab Clinical. CDM, IDC, TP and GSD declare that there are no conflicts of interest.

Consent for publication

All authors approved this manuscript for publication.

\section{Ethics approval and consent to participate}

The study protocol has been approved by the Sydney Local Health District Human Research Ethics Committee, Royal Prince Alfred Hospital, Sydney, Australia (X14-0369 \& HREC/14/RPAH/492). Any modifications to the research protocol will be notified to this human research ethics committee. Written informed consent will be obtained from participants before enrolment (see Additional file 2).

\section{Sponsor}

This research study is being sponsored by the University of Sydney, NSW, Australia, who provide indemnity.

\section{Author details}

${ }^{1}$ The Boden Institute of Obesity, Nutrition, Exercise and Eating Disorders, University of Sydney, Sydney, Australia. ${ }^{2}$ University of Sydney, Sydney Medical School, Sydney, Australia. ${ }^{3}$ Medlab Clinical, Sydney, Australia. ${ }^{4}$ School of Life and Environmental Sciences, University of Sydney, Sydney, Australia.

Received: 15 July 2016 Accepted: 20 December 2016 Published online: 09 January 2017 


\section{References}

1. Turnbaugh PJ, Ley RE, Mahowald MA, Magrini V, Mardis ER, Gordon Jl. An obesity-associated gut microbiome with increased capacity for energy harvest. Nature. 2006:444:1027-31.

2. Cani PD, Neyrinck AM, Fava F, Knauf C, Burcelin RG, Tuohy KM, Gibson GR, Delzenne NM. Selective increases of bifidobacteria in gut microflora improve high-fat-diet-induced diabetes in mice through a mechanism associated with endotoxaemia. Diabetologia. 2007;50:2374-83.

3. Ridaura VK, Faith JJ, Rey FE, Cheng J, Duncan AE, Kau AL, Griffin NW, Lombard V, Henrissat B, Bain JR, et al. Gut microbiota from twins discordant for obesity modulate metabolism in mice. Science. 2013;341:1241214.

4. Koeth RA, Wang Z, Levison BS, Buffa JA, Org E, Sheehy BT, Britt EB, Fu X, Wu Y, Li L, et al. Intestinal microbiota metabolism of L-carnitine, a nutrient in red meat, promotes atherosclerosis. Nat Med. 2013;19:576-85.

5. Vrieze A, Van Nood E, Holleman F, Salojarvi J, Kootte RS, Bartelsman JF, Dallinga-Thie GM, Ackermans MT, Serlie MJ, Oozeer R, et al. Transfer of intestinal microbiota from lean donors increases insulin sensitivity in individuals with metabolic syndrome. Gastroenterology. 2012;143:913-6. e917.

6. Cox LM, Blaser MJ. Pathways in microbe-induced obesity. Cell Metab. 2013;17:883-94

7. Zeevi D, Korem T, Zmora N, Israeli D, Rothschild D, Weinberger A, Ben-Yacov O, Lador D, Avnit-Sagi T, Lotan-Pompan M, et al. Personalized nutrition by prediction of glycemic responses. Cell. 2015;163:1079-94.

8. World Health Organization and Food and Agriculture Organization of the United Nations. Probiotics in food: Health and nutritional properties and guidelines for evaluation. Rome: Fao food and nutrition; 2006.Available at http://www.fao.org/3/a-a0512e.pdf. [Accessed Jan 2016].

9. Muccioli GG, Naslain D, Backhed F, Reigstad CS, Lambert DM, Delzenne NM, Cani PD. The endocannabinoid system links gut microbiota to adipogenesis. Mol Syst Biol. 2010;6:392.

10. Sun J, Buys NJ. Glucose- and glycaemic factor-lowering effects of probiotics on diabetes: a meta-analysis of randomised placebo-controlled trials. Br J Nutr. 2016;115(7):1167-77.

11. Asemi Z, Khorrami-Rad A, Alizadeh SA, Shakeri H, Esmaillzadeh A. Effects of synbiotic food consumption on metabolic status of diabetic patients: a double-blind randomized cross-over controlled clinical trial. Clin Nutr. 2014;33(2):198-203.

12. Ejtahed HS, Mohtadi-Nia J, Homayouni-Rad A, Niafar M, Asghari-Jafarabadi M, Mofid V, Akbarian-Moghari A. Effect of probiotic yogurt containing Lactobacillus acidophilus and Bifidobacterium lactis on lipid profile in individuals with type 2 diabetes mellitus. J Dairy Sci. 2011;94:3288-94.

13. Bukowska H, Pieczul-Mroz J, Jastrzebska M, Chelstowski K, Naruszewicz M. Decrease in fibrinogen and LDL-cholesterol levels upon supplementation of diet with Lactobacillus plantarum in subjects with moderately elevated cholesterol. Atherosclerosis. 1998;137:437-8.

14. Chan A-W, Tetzlaff JM, Altman DG, Laupacis A, Gøtzsche PC, Krleža-Jerić K, Hróbjartsson A, Mann H, Dickersin K, Berlin JA, et al. SPIRIT 2013 Statement: Defining Standard Protocol Items for Clinical Trials. Ann Intern Med. 2013;158:200-7.

15. American Diabetes Association. Diagnosis and classification of diabetes mellitus. Diabetes Care. 2011:34 Suppl 1:S62-9.

16. Matsuda M, DeFronzo RA. Insulin sensitivity indices obtained from oral glucose tolerance testing: comparison with the euglycemic insulin clamp. Diabetes Care. 1999;22:1462-70.

17. Matthews DR, Hosker JP, Rudenski AS, Naylor BA, Treacher DF, Turner RC Homeostasis model assessment: insulin resistance and $\beta$-cell function from fasting plasma glucose and insulin concentrations in man. Diabetologia. 1985;28:412-9.

18. National Health and Nutrition Examination Survey (NHaNES). Anthropometry Procedures Manual. Atlanta: Centers for Disease Control and Prevention; 2007.

19. Kiernan M, Schoffman DE, Lee K, Brown SD, Fair JM, Perri MG, Haskell WL. The Stanford Leisure-Time Activity Categorical Item (L-Cat): a single categorical item sensitive to physical activity changes in overweight/obese women. Int J Obes (Lond). 2013;37:1597-602.

20. Benjamini Y, Hochberg Y. Controlling the false discovery rate: a practical and powerful approach to multiple testing. J R Stat Soc B Methodol. 1995:57:289-300

21. Karlsson FH, Tremaroli V, Nookaew I, Bergstrom G, Behre CJ, Fagerberg B, Nielsen J, Backhed F. Gut metagenome in European women with normal, impaired and diabetic glucose control. Nature. 2013;498:99-103.
22. Napolitano A, Miller S, Nicholls AW, Baker D, Van Horn S, Thomas E, Rajpal D, Spivak A, Brown JR, Nunez DJ. Novel gut-based pharmacology of metformin in patients with type 2 diabetes mellitus. PLoS One. 2014:9:e100778.

23. Greenway F, Wang S, Heiman M. A novel cobiotic containing a prebiotic and an antioxidant augments the glucose control and gastrointestinal tolerability of metformin: a case report. Benef Microbes. 2014;5:29-32.

\section{Submit your next manuscript to BioMed Central and we will help you at every step:}

- We accept pre-submission inquiries

- Our selector tool helps you to find the most relevant journal

- We provide round the clock customer support

- Convenient online submission

- Thorough peer review

- Inclusion in PubMed and all major indexing services

- Maximum visibility for your research

Submit your manuscript at www.biomedcentral.com/submit
Biomed Central 